"Mircea cel Batran" Naval Academy Scientific Bulletin, Volume XIX - 2016 - Issue 1

Published by "Mircea cel Batran" Naval Academy Press, Constanta, Romania // The journal is indexed in: PROQUEST / DOAJ / DRJI / JOURNAL INDEX / I2OR / SCIENCE LIBRARY INDEX / Google Scholar / Crossref /

Academic Keys / ROAD Open Access / OAJI / Academic Resources / Scientific Indexing Services / SCIPIO

\title{
ABOUT THE STUDY OF THE THERMAL STRESS FOR NAVAL SYSTEMS
}

\author{
Anasase PRUIU ${ }^{1}$ \\ Beazit ALI ${ }^{2}$ \\ Ion-Adrian GIRBA ${ }^{3}$ \\ Daniel MARASESCU ${ }^{4}$ \\ ${ }^{1}$ Professor PhD Eng., Marine Engineering and Naval Weapons Departament \\ ${ }^{2}$ Professor PhD Eng., Marine Engineering and Naval Weapons Departament \\ ${ }^{3} \mathrm{PhD}$ attendee Eng., Military Tehnical Academy \\ ${ }^{4} \mathrm{PhD}$ attendee Eng., Marine Engineering and Naval Weapons Departament
}

\begin{abstract}
In this paper are presented and analyzed the effects of thermal expansion on gas evacuation piping from naval power plants and technical protection possibilities to prevent structures from deformations; also are analyzed the possibilities for the use of thermal expansion for tightening the main screws for power plant propulsion.
\end{abstract}

Keywords: compensating pipe, linear temperature expansion coefficient, overall heat exceanger temperature, thermal stress.

\section{INTRODUCTION}

In machinery, mechanisms and naval installations, mass flows of fluids circulating at different temperatures so that metal structures subject to dimensional changes. These changes are in volume:

$V=V_{0} \cdot(1+\beta \cdot \Delta t) \quad(1)$, where:

- $V_{0}\left[\mathrm{~m}^{3}\right]$ - the volume at the reference temperature;

- $V\left[\mathrm{~m}^{3}\right]$ - the volume at the heating operation;

- $\beta\left[\left({ }^{0} \mathrm{C}\right)^{-1}\right]$ - the volume expansion coefficient;

- $\Delta t[0 \mathrm{C}]$ - the temperature difference between the operating temperature and the reference temperature.

Depending on the operating temperature, the metal structures can compress or expand. Geometric reports of steel structure allow to take into account, sometimes, only by one dimension that is the direction for the study of thermal stress.

Result:

$$
L=L_{0} \cdot(1+\alpha \cdot \Delta t) \quad \text { (2), }
$$

- $L_{0}[\mathrm{~m}]$ - length at the reference temperature;

- $L[\mathrm{~m}]$ - length at mounting temperature or the operation temperature and the reference temperature;

- $\alpha\left[{ }^{0} \mathrm{C}^{-1}\right]$ - the specific linear expansion coefficient of the material from which is made the metal structure;

- $\Delta t$ - the temperature difference between the mounting temperature or the operation temperature and the reference temperature.

Equation (2) becomes:
$\Delta L=L_{0} \cdot \alpha \cdot \Delta t \quad$ (3) or:
$\frac{\Delta L}{L_{0}}=\alpha \cdot \Delta t \quad(4)$
$\sigma=\frac{\Delta L}{L_{0}} \cdot E \quad\left[\frac{\mathrm{kN}}{\mathrm{m}^{2}}\right]$
(5),

- $\sigma\left[\frac{\mathrm{kN}}{\mathrm{m}^{2}}\right]$ - tension or unit loading;

- $E\left[\frac{\mathrm{kN}}{\mathrm{m}^{2}}\right]$ - the module of longitudinal elasticity.

Temperature change will cause stress in a fixed pipe. When temperature is changed - stress introduced in a fixed pipe:

$$
\sigma=\alpha \cdot E \cdot \Delta t\left[\frac{\mathrm{kN}}{\mathrm{m}^{2}}\right] \text { (6) where: }
$$

- $\sigma-\left[\frac{\mathrm{kN}}{\mathrm{m}^{2}}\right]$ stress;

- $\alpha-\left[{ }^{\circ} \mathrm{C}^{-1}\right]$ - linear temperature expansion

coefficient

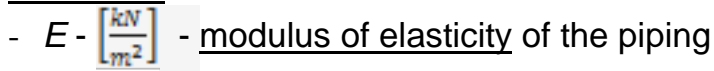
material;

- $\Delta t$ - difference temperature change from installation temperature $\left[{ }^{\circ} \mathrm{C}\right]$;

- $\Delta t=t_{i}-t_{\text {ref }}$;

- $t_{\text {ref }}$ - reference temperature;

The stress must not exceed maximum allowable stress_for the chosen piping material. Be aware that with frequently temperature changes - the stress cycle (with stress well below the maximum allowable limit) may fatigue the pipe.

Ship classification societies attach great importance of this issue. As an example, Det Norske Veritas among others, issued the general piping design for standardization like:

Stress calculation:

- When a thermal stress analysis of a piping system between two or more anchor points is carried out, the system shall be treated as a whole. The significance of all parts of the line, of restraints such as solid hangers, sway braces and guides and of intermediate restraints built in for the purpose of reducing loads on equipment or small branch lines, shall be duly considered. The stress analysis shall be carried out on the assumption 
"Mircea cel Batran" Naval Academy Scientific Bulletin, Volume XIX - 2016 - Issue 1

Published by "Mircea cel Batran" Naval Academy Press, Constanta, Romania // The journal is indexed in: PROQUEST / DOAJ / DRJI / JOURNAL INDEX / I2OR / SCIENCE LIBRARY INDEX / Google Scholar / Crossref /

Academic Keys / ROAD Open Access / OAJI / Academic Resources / Scientific Indexing Services / SCIPIO

that the piping system expands from $20^{\circ} \mathrm{C}$ to the highest operating temperature. The modulus of elasticity to be used for the pipe material, is the value of same at $20^{\circ} \mathrm{C}$.

- In carrying out a thermal stress analysis, stress concentration factors found to exist in components other than straight pipes, shall be taken into account. In cases where it is known that such components possess extra flexibility, this may be incorporated in the stress calculations. Stress concentration factors and flexibility factors given, will be accepted for use in the calculations when other substantiated factors may be lacking.

- The thermal expansion resultant stress $\sigma_{r}$ is defined as:

$\sigma_{r}=\sqrt{\sigma_{b}^{2}+4 \tau^{2}} \quad\left[\frac{N}{m m^{2}}\right]$

$\sigma_{b}=\frac{\sqrt{\left(\mathrm{i}_{1} M_{1}\right)^{2}+\left(\mathrm{i}_{0} M_{0}\right)^{2}}}{2} \quad\left[\frac{N}{m^{2}}\right]$ (8), represent total bending stress;

$\tau=\frac{M_{T}}{2 z}\left[\frac{\mathrm{N}}{\mathrm{mm}^{2}}\right]$, represented torsional stress,

$\mathrm{M}_{T}-$ torsion moment [Nm],

$M_{l}$ - bending moment in plane of member [Nm];

Mo-bending moment traverse to plane of member [Nm];

$i_{1}$ - stress concentration factor for in-plane bendingmoments;

io - stress concentration factor for out-of-plane bending moments;

$Z=$ section modulus in bending of member $\left(\mathrm{mm}^{3}\right)$. When the member cross-section in non-uniform, the section modulus of the matching pipe shall be used. For branched systems, where the branch diameter is less than the header diameter, the branch section modulus may be taken as the smaller value of:

$$
\pi n_{b}^{2} t_{h} \text { and } \pi n_{b}^{2} i_{i b} t_{b}
$$

$r_{b}$ - mean cross-sectional radius of branch [mm]

$t_{h}$ - thickness of pipe which matches header [mm]

$t_{b}$ - thickness of pipe which matches branch [mm]

$\mathrm{I}_{\text {ib }}$ - in-plane stress concentration factor for branch.

The resultant stress $\sigma_{r}$ is at no point of the piping system to exceed the corresponding stress range $\sigma_{\text {int }}$ : $\sigma_{\text {int }}=0.75 \sigma_{\text {tk }}+0.25 \sigma_{\text {tv }}$

$\sigma_{t k_{-}}$permissible pipe wall stress at $100^{\circ} \mathrm{C}$ or lower

$\left[\frac{N}{m m^{2}}\right]$;

$\sigma_{t v}$ - permissible pipe wall stress at maximum working temperature of system $\left[\frac{\mathrm{N}}{\mathrm{mm}^{2}}\right]$;

For low temperature piping $\sigma_{\text {int }}$ shall be determined upon special consideration.

The sum of axial bending stress in the pipe wall due to static loading (pipe weight) and axial tensile stress

due to internal pressure, is at no point in the system to exceed the permissible stress $\sigma_{t v}$.

Table 1-Linear Temperature Expansion Coefficient [a]

\begin{tabular}{|l|l|}
\hline $\begin{array}{l}\text { Linear Temperature Expansion } \\
\text { Coefficient [a] }\end{array}$ & {$\left[{ }^{0} \mathrm{C}^{-1}\right]$} \\
\hline Brass & $18,7 \cdot 10^{-6}$ \\
\hline $\begin{array}{l}\text { Epoxy, cast resins \& } \\
\text { compounds, unfilled }\end{array}$ & $(45-65) \cdot 10^{-6}$ \\
\hline Invar & $1,5 \cdot 10^{-6}$ \\
\hline Nickel & $13 \cdot 10^{-6}$ \\
\hline Steel & $12 \cdot 10^{-6}$ \\
\hline Steel Stainless Austenitic (304) & $17,3 \cdot 10^{-6}$ \\
\hline Steel Stainless Austenitic (310) & $14,4 \cdot 10^{-6}$ \\
\hline Steel Stainless Austenitic (316) & $16 \cdot 10^{-6}$ \\
\hline Steel Stainless Ferritic (410) & $9,9 \cdot 10^{-6}$ \\
\hline Carbon steel pipes & $11,6 \cdot 10^{-6}$ \\
\hline Austenitic steel pipes & $13,5 \cdot 10^{-6}$ \\
\hline
\end{tabular}

Table 2- Tensile Modulus

\begin{tabular}{|l|l|}
\hline Tensile Modulus [E] & {$\left[\mathrm{kN} / \mathrm{m}^{2}\right]$} \\
\hline Brass & $1,02 \cdot 10^{8}$ \\
\hline Copper & $1,17 \cdot 10^{8}$ \\
\hline Epoxy, cast resins \& & $(0,02-0,03)$ \\
compounds, unfilled & $-10^{8}$ \\
\hline Nickel & $1,7 \cdot 10^{8}$ \\
\hline Steel, stainless ANSI 302 & $1,8 \cdot 10^{8}$ \\
\hline Steel, Structural ASTM-A36 & $2 \cdot 10^{8}$ \\
\hline
\end{tabular}

Table 3 - International standards for ambient reference conditions

$25^{\star}$ - charge air coolant temperature;

$10^{\star \star}$ - cooling water temperature (minimum for lubricating oil cooler). 
"Mircea cel Batran" Naval Academy Scientific Bulletin, Volume XIX - 2016 - Issue 1

Published by "Mircea cel Batran" Naval Academy Press, Constanta, Romania // The journal is indexed in: PROQUEST / DOAJ I DRJI / JOURNAL INDEX I I2OR / SCIENCE LIBRARY INDEX / Google Scholar / Crossref I Academic Keys / ROAD Open Access / OAJI / Academic Resources / Scientific Indexing Services / SCIPIO

\section{A. AXIAL FORCE THAT EMERGED FROM TEMPERATURE DIFFERENCE}

Axial force developed into a pipe due to thermal expansion or thermal compression:

$$
F=\sigma_{\mathrm{t}} \cdot E \cdot \Delta t \cdot S_{u} \quad(10),
$$

$S_{u}$ - surface of the pipe section $\left[\mathrm{m}^{2}\right] ;$

$S_{u}=\frac{\pi}{4}\left(d_{i}^{2}-d_{i}^{2}\right)\left[\mathrm{m}^{2}\right]$, where:

$d_{e}$ - the outer diameter of the pipe [m];

$d_{i}$ - the inner diameter of the pipe [m];

$s=\frac{d_{x}-d_{i}}{2}[\mathrm{~m}](11)$, the pipe thickness;

$d_{m}=\frac{d_{i}-d_{g}}{2}[\mathrm{~m}]$ (12), the average diameter of the piupe.

Table 4 are determined the forces at different values of pipe heating. It is noted that, although the temperature differences are small, flat expansion pipes are required to avoid altering the geometry of the metal structure.

Table 4 - The axial force developed in the pipe by the thermal expansion.

\begin{tabular}{|c|c|c|c|c|c|c|}
\hline $\mathrm{E}\left[\mathrm{kN} / \mathrm{m}^{2}\right]$ & $2 \cdot 10^{8}$ & $\begin{array}{c}2 \cdot \\
10^{8}\end{array}$ & $\begin{array}{c}2 \cdot \\
10^{8}\end{array}$ & $\begin{array}{c}2 \\
\cdot 10^{8}\end{array}$ & $\begin{array}{c}2 \\
\cdot 10^{8}\end{array}$ & $\begin{array}{c}2 \\
10^{8}\end{array}$ \\
\hline$[\mathrm{\alpha}]\left[\left({ }^{\circ} \mathrm{C}\right)^{-1}\right]$ & $\begin{array}{c}12 \cdot 10^{-} \\
6\end{array}$ & $\begin{array}{c}12 \\
\cdot 10^{-6}\end{array}$ & $\begin{array}{c}12 \\
\cdot 10^{-6}\end{array}$ & $\begin{array}{c}12 \\
\cdot 10^{-6}\end{array}$ & $\begin{array}{c}12 \cdot \\
10^{-6}\end{array}$ & $\begin{array}{c}12 \\
\cdot 10^{-6}\end{array}$ \\
\hline $\mathrm{t}_{0}\left[{ }^{0} \mathrm{C}\right] \mathrm{ISO}$ & 25 & 25 & 25 & 25 & 25 & 25 \\
\hline $\mathrm{t}_{\mathrm{i}}\left[{ }^{0} \mathrm{C}\right]$ & 30 & 35 & 40 & 45 & 50 & 55 \\
\hline $\mathrm{dt}\left[{ }^{0} \mathrm{C}\right]$ & 5 & 10 & 15 & 20 & 25 & 30 \\
\hline$[\sigma]\left[\mathrm{kN} / \mathrm{m}^{2}\right]$ & $\begin{array}{c}120 \cdot 10 \\
2\end{array}$ & $\begin{array}{c}240 \cdot \\
10^{2}\end{array}$ & $\begin{array}{c}360 \cdot \\
10^{2}\end{array}$ & $\begin{array}{c}480 \cdot \\
10^{2}\end{array}$ & $\begin{array}{c}600 \cdot \\
10^{2}\end{array}$ & $\begin{array}{c}720 \\
10^{2}\end{array}$ \\
\hline$[\sigma][\mathrm{bar}]$ & 120 & 240 & 360 & 480 & 600 & 720 \\
\hline $\mathrm{Su}\left[\mathrm{m}^{2}\right]$ & $10^{-2}$ & $10^{-2}$ & $10^{-2}$ & $10^{-2}$ & $10^{-2}$ & $10^{-2}$ \\
\hline $\mathrm{F}[\mathrm{kN}]$ & 120 & 240 & 360 & 480 & 600 & 720 \\
\hline $\mathrm{F}\left[\mathrm{t}_{\mathrm{f}}\right]$ & 12,18 & $\begin{array}{c}24,3 \\
6\end{array}$ & $\begin{array}{c}36,5 \\
5\end{array}$ & $\begin{array}{c}48,7 \\
3\end{array}$ & 61 & 73 \\
\hline
\end{tabular}

Obs. Pipe $d_{m}=250[\mathrm{~mm}] ; s=12.73[\mathrm{~mm}] ; S_{u}=10$ ${ }^{2}\left[m^{2}\right]$

\section{B. TIGHTENING OF THE MAIN SCREWS}

Main screws are considered: tie-rods for assembling the heat engine, engine mounting bolts on the base or supporting structure of the ship, connecting rod big end bolts to assemble the head and the cylinder head bolts.

Determine the clamping force:

$F_{s t}=(2 \div 3) F_{\text {dim }} \quad[\mathrm{kN}] \quad(13)$,

$F_{\text {dim }}[\mathrm{kN}]$-sizing force.

The clamping force can be obtained by the following methods:

\begin{tabular}{|c|c|c|c|c|}
\hline $\begin{array}{l}\text { International standards } \\
\text { for ambient reference } \\
\text { conditions }\end{array}$ & $\begin{array}{l}\mathbf{t} \text { air } \\
{\left[{ }^{0} \mathrm{C}\right]}\end{array}$ & $\begin{array}{c}\mathbf{p} \\
{[\mathrm{bar}]}\end{array}$ & $\begin{array}{l}\text { Humidiy } \\
{[\%]}\end{array}$ & $\begin{array}{l}\mathrm{t}_{\mathrm{sw}} \\
{\left[{ }^{0} \mathrm{C}\right]}\end{array}$ \\
\hline $\begin{array}{lr}\text { STP - } & \text { Standard } \\
\text { Temperature } & \text { and } \\
\text { Pressure } & \text { IUPAC } \\
\text { (International } & \text { Union of } \\
\text { Pure and } & \text { Applied } \\
\text { Chemistry) } & \end{array}$ & 0 & 1 & & \\
\hline $\begin{array}{lr}\text { STP - } & \text { Standard } \\
\text { Temperature } & \text { and } \\
\text { Pressure USA } & \end{array}$ & 15,6 & 1,0132 & & \\
\hline $\begin{array}{lr}\text { NTP }- & \text { Normal } \\
\text { Temperature } & \text { and } \\
\text { Pressure } & \end{array}$ & 20 & 1,0132 & & \\
\hline $\begin{array}{l}\text { SATP - Standard Ambient } \\
\text { Temperature } \\
\text { Pressure }\end{array}$ & 25 & 1,0132 & & \\
\hline $\begin{array}{l}\text { ISA - International } \\
\text { Standard Atmosphere }\end{array}$ & 15 & 1,0132 & 0 & \\
\hline $\begin{array}{l}\text { ICAO Standard } \\
\text { Atmosphere }\end{array}$ & 15 & 1,0132 & & \\
\hline $\begin{array}{l}\text { ISO 3046-1:2002(E);ISO } \\
15550: 2002(\mathrm{E})\end{array}$ & 25 & 1 & 30 & $25^{*}$ \\
\hline $\begin{array}{l}\text { IACS M28(1978): Tropical } \\
\text { ambient } \\
\text { conditions }\end{array}$ & 45 & 1 & 60 & 32 \\
\hline $\begin{array}{l}\text { Winter ambient reference } \\
\text { conditions }\end{array}$ & 10 & 1 & 60 & $10^{* *}$ \\
\hline
\end{tabular}

- hydraulic devices for bolt elongation<

- elongation of the screw by turning the nut with torque wrench;

- bolt elongation with star key with beating;

- bolt elongation by thermal expansion, electrical resistance being mounted in the main bolt .

For check assembly- tightening of main screw , the following methods are used:

- measuring the pressure in the hydraulic device;

- bolt elongation measurement;

- measuring the angle of rotation of the nut;

- measuring the temperature of the heating screw thereof.

$F_{s t}=\sigma_{s t} \cdot A_{g}[\mathrm{kN}](14) ;$

$\sigma_{s t}=\xi_{s t} \cdot E\left[\frac{N}{m^{2} m^{2}}\right]$ (15), where:

$\xi_{s t}=\frac{\Delta L_{s t}}{L_{0}} \quad[-]-$ the relative elongation of the bolt;

$\Delta L_{\text {st }}[\mathrm{mm}]$ - the actual elongation of the bolt;

$L_{0}[\mathrm{~mm}]$ - length of the screw at the reference temperature;

$F_{s t}=\frac{\Delta L_{s t}}{L_{0}} \cdot E \cdot A_{s} \quad[\mathrm{kN}](16) ;$

$\sigma_{s t}=\frac{\Delta L_{s t}}{L_{0}} \cdot E\left[\frac{N}{m m^{2}}\right]$

$\sigma_{s t}=\alpha \cdot E \cdot \Delta t\left[\frac{N}{m m^{2}}\right] ;$ 
"Mircea cel Batran" Naval Academy Scientific Bulletin, Volume XIX - 2016 - Issue 1

Published by "Mircea cel Batran" Naval Academy Press, Constanta, Romania // The journal is indexed in: PROQUEST / DOAJ I DRJI / JOURNAL INDEX I I2OR / SCIENCE LIBRARY INDEX / Google Scholar / Crossref I

Academic Keys / ROAD Open Access / OAJI / Academic Resources / Scientific Indexing Services / SCIPIO

$\Delta t=\frac{1}{\alpha} \cdot \frac{\Delta L_{s t}}{L}, \quad\left[{ }^{0} \mathrm{C}\right](17)$, determined in Table 5.

Table 5- Tightening the main screws, by thermal expansion.

\begin{tabular}{|c|c|c|c|c|}
\hline & $\begin{array}{c}\text { Studs } \\
\text { fixing } \\
\text { pedesta } \\
\text { | }\end{array}$ & $\begin{array}{l}\text { Connecting } \\
\text { rod bolt head }\end{array}$ & $\begin{array}{c}\text { Cylinde } \\
\text { r head } \\
\text { bolt }\end{array}$ & $\begin{array}{c}\text { Couplin } \\
\text { g } \\
\text { bar }\end{array}$ \\
\hline $\mathrm{L}[\mathrm{mm}]$ & 500 & 1500 & 1200 & 9000 \\
\hline $\mathrm{dl}[\mathrm{mm}]$ & $\begin{array}{c}0,25- \\
0,35\end{array}$ & $0,65-1,05$ & $0,6-0,84$ & $4,5-6,3$ \\
\hline $\mathrm{dl} / \mathrm{L}$ & $(5-7) \cdot 10^{-}$ & $(4,3-7) \cdot 10^{-4}$ & $(5-7)_{4} \cdot 10^{-}$ & $(5-7)_{4}^{\cdot 10}$ \\
\hline $\mathrm{E}\left[\mathrm{kN} / \mathrm{m}^{2}\right]$ & $2 \cdot 10^{8}$ & $2 \cdot 10^{8}$ & $2 \cdot 10^{8}$ & $2 \cdot 10^{8}$ \\
\hline$[\alpha]\left[\left({ }^{0} \mathrm{C}\right)^{-1}\right]$ & $13 \cdot 10^{-6}$ & $13 \cdot 10^{-6}$ & $13 \cdot 10^{-6}$ & $13^{-6}$ \\
\hline $\mathrm{dt}\left[{ }^{0} \mathrm{C}\right]$ & $39-54$ & $33-54$ & $39-54$ & $39-54$ \\
\hline $\begin{array}{c}\mathrm{t}_{0}\left[{ }^{0} \mathrm{C}\right] \\
\mathrm{ISO}\end{array}$ & 25 & 25 & 25 & 25 \\
\hline $\mathrm{t}_{\mathrm{i}}\left[{ }^{0} \mathrm{C}\right]$ & $64-79$ & $58-79$ & $64-79$ & $64-79$ \\
\hline
\end{tabular}

$\mathrm{t}_{\mathrm{i}}\left[{ }^{0} \mathrm{C}\right]$ - Heating temperature; $\mathrm{t}_{0}=\mathrm{t}_{\text {ref }}\left[{ }^{0} \mathrm{C}\right]=25^{\circ} \mathrm{C}$ ISO 3046-1:2002(E);ISO 15550:2002(E)

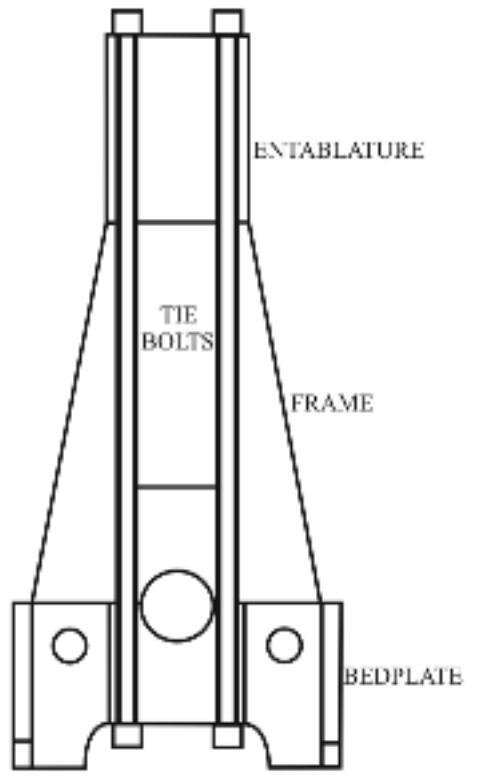

Figure1a - Coupling Bars [2.pg. 42]

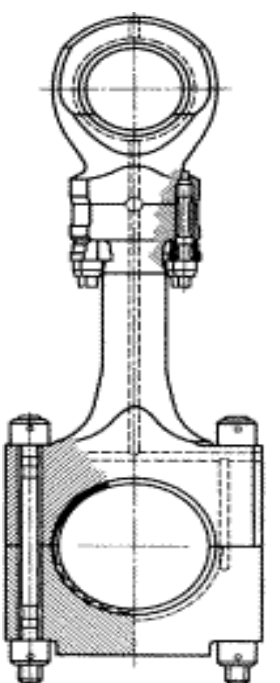

Figure $1 b$ - Join pin for crank end of connectingrod

[2, pg. 84]

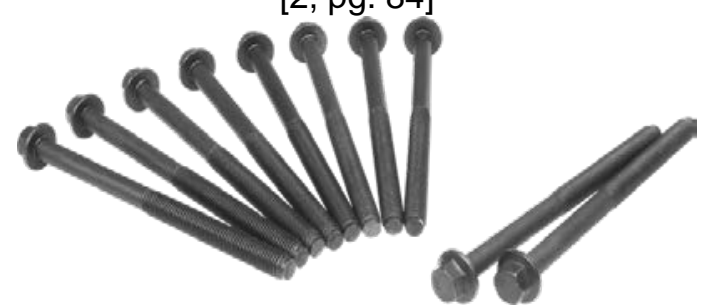

Figure 1c- Cylinder head bolts [3]

TIE BOLT TIGHTENING SEQUENCES
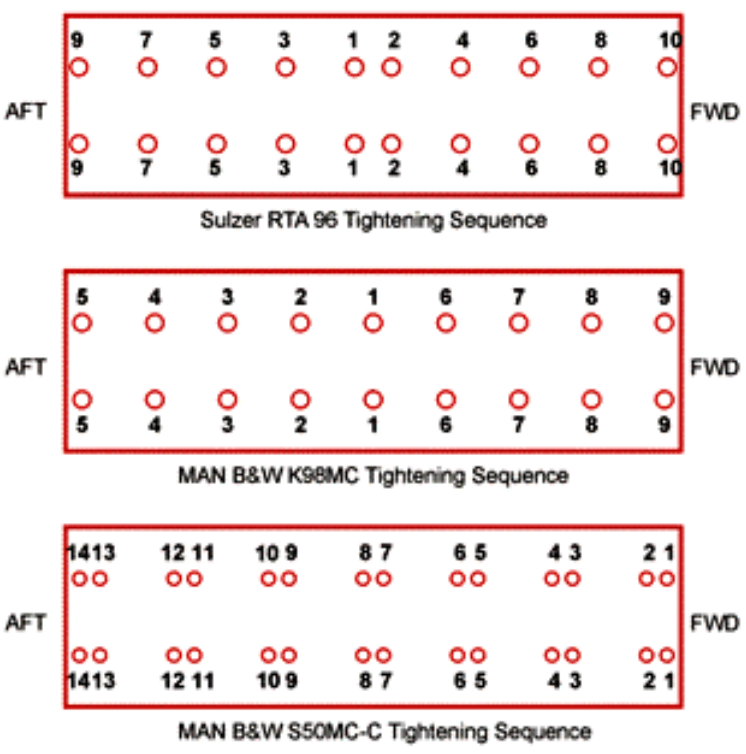

Figure 2 - The bolt tightening sequences [2, pg.44]

\section{FIXING THE MAIN ENGINE ON THE PEDESTAL}

At the coupling of the engine with shaft line it is mounts thrust bearing, which requires that the first 
"Mircea cel Batran" Naval Academy Scientific Bulletin, Volume XIX - 2016 - Issue 1

Published by "Mircea cel Batran" Naval Academy Press, Constanta, Romania /I The journal is indexed in: PROQUEST / DOAJ / DRJI / JOURNAL INDEX / I2OR / SCIENCE LIBRARY INDEX / Google Scholar / Crossref /

Academic Keys / ROAD Open Access / OAJI / Academic Resources / Scientific Indexing Services / SCIPIO

studs in the aft, which fix the engine on the pedestal, be calibrated on the holes in the engine flange and in the holes in the pedestal, and the holes from the forward of engine not be clearance so as not to allow the engine, expansion, In view of the temperatures of the motor flange, respectively the flange of the pedestal, in Table 6 are determined clearances required for assembly, to avoid blocking the engine longitudinal expansion and its cracking in the area of engine frame and block cylinders.

Table 6 - Dilation of the engine compared to the base structure of the ship

\begin{tabular}{|l|l|l|l|l|l|}
\hline $\begin{array}{l}{\left[\alpha_{\mathrm{FM}}\right]} \\
{\left[\left({ }^{\circ} \mathrm{C}\right)^{-1}\right]}\end{array}$ & $13 \cdot 10^{-6}$ & $13 \cdot 10^{-6}$ & $13 \cdot 10^{-6}$ & $13 \cdot 10^{-6}$ & $13 \cdot 10^{-6}$ \\
\hline $\begin{array}{l}{\left[\alpha_{\mathrm{FP}}\right]} \\
\left.\left[{ }^{0} \mathrm{C}\right)^{-1}\right]\end{array}$ & $12 \cdot 10^{-6}$ & $12 \cdot 10^{-6}$ & $12 \cdot 10^{-6}$ & $12 \cdot 10^{-6}$ & $12 \cdot 10^{-6}$ \\
\hline $\begin{array}{l}\mathrm{L}_{0} \\
{[\mathrm{~mm}]}\end{array}$ & $10^{4}$ & $10^{4}$ & $10^{4}$ & $10^{4}$ & $10^{4}$ \\
\hline $\begin{array}{l}\mathrm{t}_{0}\left[{ }^{0} \mathrm{C}\right] \\
\text { ISO }\end{array}$ & 25 & 25 & 25 & 25 & 25 \\
\hline $\mathrm{t}_{\mathrm{FM}}\left[{ }^{0} \mathrm{C}\right]$ & 10 & 20 & 25 & 30 & 35 \\
\hline $\begin{array}{l}\Delta \mathrm{t}_{\mathrm{FM}} \\
{\left[{ }^{0} \mathrm{C}\right]}\end{array}$ & -15 & -5 & 0 & +5 & +10 \\
\hline $\mathrm{t}_{\mathrm{FP}}\left[{ }^{0} \mathrm{C}\right]$ & 10 & 20 & 20 & 20 & 20 \\
\hline$\Delta \mathrm{t}_{\mathrm{FP}}\left[{ }^{0} \mathrm{C}\right]$ & -15 & -5 & -5 & -5 & -5 \\
\hline $\begin{array}{l}\Delta \mathrm{L}_{\mathrm{FM}} \\
{[\mathrm{mm}]}\end{array}$ & $-1,95$ & $-0,65$ & 0 & $+0,65$ & $+1,3$ \\
\hline $\begin{array}{l}\Delta \mathrm{L}_{\mathrm{FP}} \\
{[\mathrm{mm}]}\end{array}$ & $-1,80$ & $-0,6$ & $-0,6$ & $-0,6$ & $-0,6$ \\
\hline $\begin{array}{l}\sum \Delta \mathrm{L} \\
{[\mathrm{mm}]}\end{array}$ & $+0,15$ & $+0,05$ & $-0,6$ & $-1,25$ & $-1,9$ \\
\hline
\end{tabular}

\section{STEAM PIPING EXPANSION}

Table 7 - Saturated Steam Piping and Superheated Steam Piping

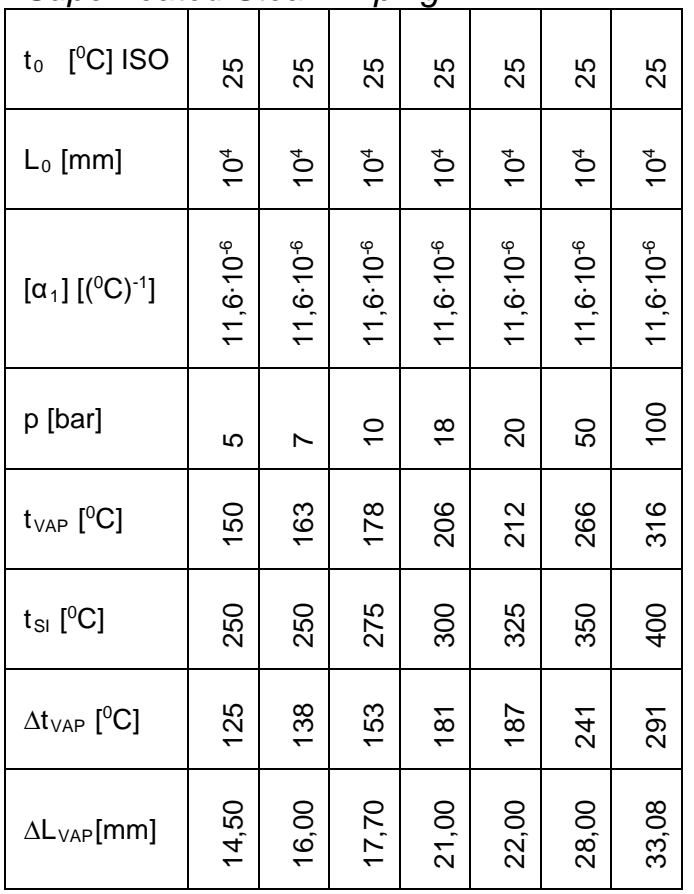

DOI: 10.21279/1454-864X-16-I1-048

(C) 2015. This work is licensed under the Creative Commons Attribution-Noncommercial-Share Alike 4.0 License.

\begin{tabular}{|c|c|c|c|c|c|c|c|}
\hline$\Delta \mathrm{t}_{\mathrm{SI}}\left[{ }^{0} \mathrm{C}\right]$ & $\underset{\sim}{\stackrel{\sim}{\sim}}$ & $\stackrel{\sim}{N}$ & 怘 & 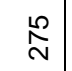 & ঃ্লি & $\stackrel{\sim}{\tilde{N}}$ & 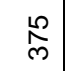 \\
\hline$\Delta \mathrm{L}_{\mathrm{sI}}[\mathrm{mm}]$ & $\begin{array}{l}m \\
\stackrel{D}{N}\end{array}$ & $\stackrel{-1}{\hat{\sigma}^{-}}$ & 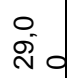 & $\stackrel{\circ}{\tilde{m}} \underset{0}{0}$ & $\begin{array}{l}\infty \\
\tilde{m}^{-}\end{array}$ & $\hat{\tilde{m}}$ & $\begin{array}{l}0 \\
\stackrel{\tilde{F}}{\sigma}\end{array}$ \\
\hline
\end{tabular}

E. The Gas Piping Expansion, To Internal Combustion Engines

Table 8 - The gas piping of internal combustion

\begin{tabular}{|c|c|c|c|c|c|c|}
\hline \multicolumn{7}{|c|}{ engines } \\
\hline $\mathrm{t}_{0}\left[{ }^{\circ} \mathrm{C}\right]$ ISO & $\stackrel{\mathscr{N}}{\sim}$ & $\stackrel{\llcorner}{N}$ & $\stackrel{\mathscr{N}}{\sim}$ & $\stackrel{\mathscr{N}}{\sim}$ & $\stackrel{\llcorner}{\sim}$ & $\stackrel{\mathscr{N}}{\sim}$ \\
\hline $\mathrm{t}_{\mathrm{EV}} \quad\left[{ }^{\circ} \mathrm{C}\right]$ & 品 & \& & 多 & \& & 吕 & : \\
\hline$\left.\left[\alpha_{2}\right]\left[{ }^{(} \mathrm{C}\right)^{-1}\right]$ & 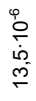 & $\begin{array}{l}\dot{0} \\
\stackrel{0}{\dot{b}} \\
\stackrel{\rho}{r} \\
\dot{p}\end{array}$ & $\begin{array}{l}\stackrel{0}{0} \\
\dot{0} \\
\dot{\rho} \\
\stackrel{\rho}{2}\end{array}$ & $\begin{array}{l}\stackrel{0}{0} \\
\dot{5} \\
\stackrel{\rho}{\sigma}\end{array}$ & $\begin{array}{l}0 \\
\dot{0} \\
\dot{\rho} \\
\stackrel{p}{-}\end{array}$ & $\begin{array}{l}\stackrel{0}{\circ} \\
\stackrel{0}{\circ} \\
\stackrel{\rho}{\Gamma}\end{array}$ \\
\hline$\Delta \mathrm{t}\left[{ }^{\circ} \mathrm{C}\right]$ & $\stackrel{\text { N్ల }}{\text { N }}$ & 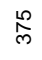 & $\stackrel{\stackrel{N}{\sim}}{ }$ & 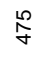 & $\stackrel{\sim ్ N}{\sim ్}$ & 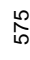 \\
\hline $\mathrm{L}_{0}[\mathrm{~mm}]$ & 苛 & 苛 & 苛 & 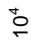 & 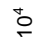 & 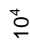 \\
\hline$\Delta \mathrm{L}[\mathrm{mm}]$ & $\begin{array}{l}\infty \\
\infty \\
\tilde{g}\end{array}$ & $\begin{array}{l}8 \\
0 \\
0\end{array}$ & $\stackrel{\infty}{\stackrel{m}{\hbar}}$ & \begin{tabular}{l}
$m$ \\
\multirow{0}{0}{}
\end{tabular} & i & 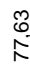 \\
\hline
\end{tabular}

\section{F. THE EXPANSION OF GAS PIPING FROM} STEAM BOILERS

Table 9 - The gas piping - burner steam boiler

\begin{tabular}{|c|c|c|c|c|c|c|}
\hline $\begin{array}{c}\mathrm{t}_{0}\left[{ }^{0} \mathrm{C}\right] \\
\mathrm{ISO}\end{array}$ & 25 & 25 & 25 & 25 & 25 & 25 \\
\hline $\begin{array}{c}\mathrm{t}_{\mathrm{EV}} \\
{\left[{ }^{\circ} \mathrm{C}\right]}\end{array}$ & 125 & 150 & 175 & 200 & 225 & 250 \\
\hline$\Delta \mathrm{t}\left[{ }^{0} \mathrm{C}\right]$ & 100 & 125 & 150 & 175 & 200 & 225 \\
\hline $\begin{array}{c}\mathrm{L}_{0} \\
{[\mathrm{~mm}]}\end{array}$ & $10^{4}$ & $10^{4}$ & $10^{4}$ & $10^{4}$ & $10^{4}$ & $10^{4}$ \\
\hline $\begin{array}{c}\Delta \mathrm{L} \\
{[\mathrm{mm}]}\end{array}$ & 13,50 & 16.88 & 20,25 & 24,00 & 27,00 & 30,38 \\
\hline
\end{tabular}

\section{G. THE EXPANSION OF GAS PIPING FROM GAS TURBINES}

Table 10 - The gas piping from gas turbines 
"Mircea cel Batran" Naval Academy Scientific Bulletin, Volume XIX - 2016 - Issue 1

Published by "Mircea cel Batran" Naval Academy Press, Constanta, Romania // The journal is indexed in: PROQUEST / DOAJ I DRJI / JOURNAL INDEX I I2OR / SCIENCE LIBRARY INDEX / Google Scholar / Crossref I

Academic Keys / ROAD Open Access / OAJI / Academic Resources / Scientific Indexing Services / SCIPIO

\begin{tabular}{|c|c|c|c|c|c|c|}
\hline $\begin{array}{c}\mathrm{t}_{0} \\
{\left[{ }^{\circ} \mathrm{C}\right]} \\
\mathrm{ISO}\end{array}$ & 25 & 25 & 25 & 25 & 25 & 25 \\
\hline $\begin{array}{c}\mathrm{t}_{\mathrm{EV}} \\
{\left[{ }^{\circ} \mathrm{C}\right]}\end{array}$ & 525 & 550 & 600 & 625 & 700 & 750 \\
\hline $\begin{array}{c}\Delta \mathrm{t} \\
{\left[{ }^{\circ} \mathrm{C}\right]}\end{array}$ & 500 & 525 & 575 & 600 & 675 & 725 \\
\hline $\begin{array}{c}\mathrm{L}_{0} \\
{[\mathrm{~mm}]}\end{array}$ & $10^{4}$ & $10^{4}$ & $10^{4}$ & $10^{4}$ & $10^{4}$ & $10^{4}$ \\
\hline $\begin{array}{c}\Delta \mathrm{L} \\
{[\mathrm{mm}]}\end{array}$ & 67,50 & 71,00 & 77,63 & 81,00 & 91,13 & 98,0 \\
\hline
\end{tabular}

Observation - Compensating pipe shown in figure $3 a, b, c$.

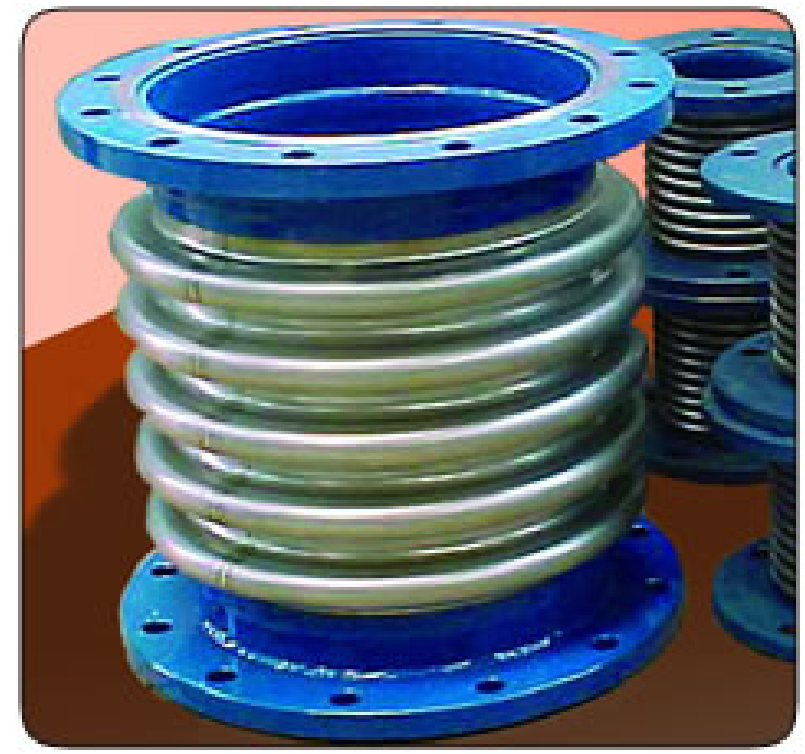

a)

Compensating pipe for flue gas exhaust ducts [4]

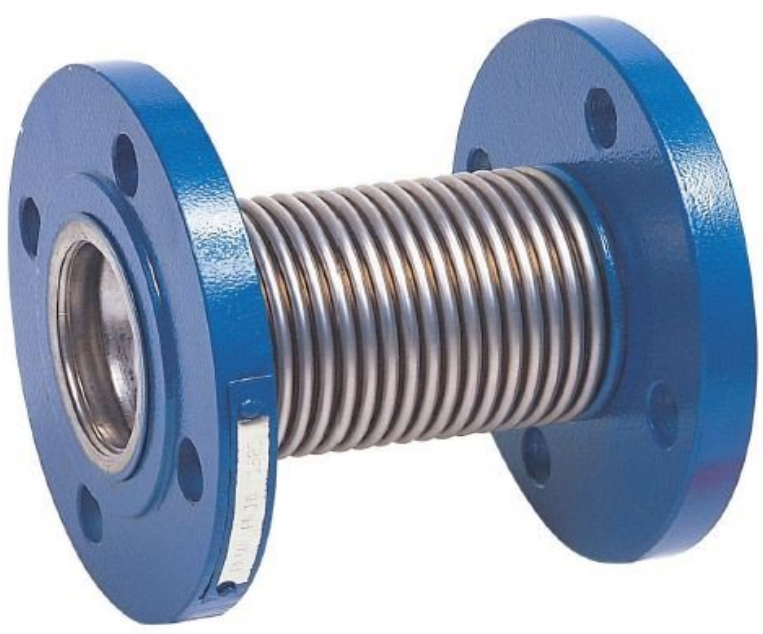

a) Compensating pipe for The gas piping burner steam boiler [4]

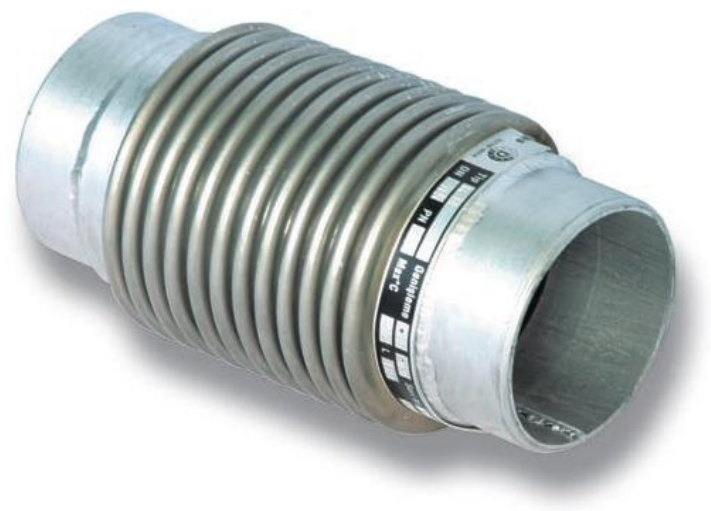

b) axial compensating pipe for linear expansion [5]

Figure 3 - Dilatation compensating pipe

\section{CONCLUSIONS}

Particular attention should be given to fixing metal structures to enable the processing of dimensional changes. In design and installation must be determined sense of expansion to maintain safety in operation. In operational the procedures to preheating, startup and keep the operating temperatures must be respected. Attention should be given to the heating main bolts fortheir dismanting!.

\section{BIBLIOGRAPHY}

[1] Det Norske Veritas , "MARINE AND MACHINERY SYSTEMS AND EQUIPMENT", Chapter 2, Section2, C-Design Condition, October 2010;

[2] Marko Valcic-Pomorscki fakultet Rijeska „The Learning Resource for Marine Engineers”, www.

Marinediesels.co.uk;

[3] https://www.google.ro/search?q=cylinders+head+double-

ended+bolt+on+++two+stroke+marine+engine \&espv=2\&biw=1280\&bih=852\&source=Inms\&tbm=isch \&sa=X \&ved=0ahUKEwjS74Se_bHMAhXKJJoKHQnDDdIQ_AUIBigB\#tbm=isch\&q=+cylinder+head+bolts+tightenin g+on+four+stroke+marine+engine+drawing\&imgrc=buTz7EbgnPBGGM\%3A;

[4] http://www.microplasma.ro/produse_ind_lenticulari.htm; 
"Mircea cel Batran" Naval Academy Scientific Bulletin, Volume XIX - 2016 - Issue 1

Published by "Mircea cel Batran" Naval Academy Press, Constanta, Romania /I The journal is indexed in: PROQUEST / DOAJ / DRJI / JOURNAL INDEX / I2OR / SCIENCE LIBRARY INDEX / Google Scholar / Crossref / Academic Keys I ROAD Open Access / OAJI / Academic Resources / Scientific Indexing Services / SCIPIO

[5] https://www.prestcom-instal.ro/fdumi_mag/eshop/1-1-ROBINETI-INDUSTRIALI-DUYAR/50-2COMPENSATOR-AXIAL-DUYAR;

[4] http://www.engineeringtoolbox.com/linear-expansion-coefficients-d_95.html;

[6] Publications of Indian Register of Shipping, 2014 Rules and Regulations for the Construction and Classification of Steel Ships;

[7] Pounder's Marine Diesel Engines and Gas Turbines, Eighth edition, 2004. 